\title{
Chemical Imaging with Frequency Modulation Coherent Anti-Stokes Raman Scattering Microscopy at the Vibrational Fingerprint Region
}

\author{
Bi-Chang Chen, Jiha Sung, and Sang-Hyun Lim* \\ Department of Chemistry and Biochemistry, University of Texas at Austin, 1 University Station A5300, \\ Austin, Texas 78712, United States
}

Received: May 18, 2010; Revised Manuscript Received: October 25, 2010

\begin{abstract}
We present a new coherent anti-Stokes Raman scattering (CARS) method that can perform background-free microscopy and microspectroscopy at the vibrational fingerprint region. Chirped broad-band pulses from a single Ti:sapphire laser generate CARS signals over $800-1700 \mathrm{~cm}^{-1}$ with a spectral resolution of $20 \mathrm{~cm}^{-1}$. Fast modulation of the time delay between the pump and Stokes pulses coupled with lock-in signal detection not only removes the nonresonant background but also produces Raman-like CARS signals. Chemical imaging and microspectroscopy are demonstrated with various samples such as edible oils, lipid membranes, skin tissue, and plant cell walls. Systematic studies of the signal generation mechanism and several fundamental aspects are discussed.
\end{abstract}

\section{Introduction}

Over the past decade, coherent anti-Stokes Raman scattering (CARS) microscopy has developed into a powerful label-free nonlinear optical imaging technique. ${ }^{1}$ With its intrinsic vibrational contrast, great sensitivity, and three-dimensional sectioning ability, CARS microscopy has become an important labelfree chemical imaging tool in biology and material sciences. ${ }^{1-3}$ Most CARS microscopy techniques demonstrated so far have employed two synchronized picosecond laser pulses. Since vibrational response of typical organic molecules lasts a few picoseconds, laser pulses with similar time duration are optimal in the consideration of both spectral resolution and signal sensitivity. ${ }^{1,4,5}$ The use of picosecond laser pulses also reduces the nonresonant background and nonlinear photodamages significantly. ${ }^{4}$ This configuration of CARS microscopy, which we will call "narrow CARS" method from now on, has proven to be an excellent imaging tool for visualizing lipid-rich structures in cells and tissues when it detects CARS signals at $2840 \mathrm{~cm}^{-1}$.

The vibrational fingerprint region $\left(800-1800 \mathrm{~cm}^{-1}\right)$ is an important frequency range where many molecular functional groups have unique vibrational resonances. ${ }^{6}$ In this frequency region, however, the vibrational spectrum is often crowded with multiple peaks, and a single vibrational frequency peak is not enough to identify local chemical structures in many situations. ${ }^{7-10}$ In addition, the ubiquitous nonresonant background interferes with relatively weak vibrationally resonant CARS signals to distort the frequency response of measured signals significantly at this frequency region. In the past decade, there have been numerous CARS methods developed to remove the effect of nonresonant background and extract vibrational CARS signals. ${ }^{1,11-15}$ Among them, the fast frequency modulation (FM) CARS technique has proven to be an effective way to eliminate the nonresonant background. It relies on the different spectral shapes of the resonant CARS signals and nonresonant backgrounds. ${ }^{13,15}$ The line width of vibrational peaks in the fingerprint region is typically $5-20 \mathrm{~cm}^{-1}$, while the nonresonant background is spectrally flat due to the instantaneous time response of an offresonant electronic four-wave-mixing process. If one can

\footnotetext{
* Corresponding author. E-mail: shlim@mail.utexas.edu.
}

measure the difference between CARS signals at two vibrational excitation frequencies, the contribution of the nonresonant background in the measured signals can be removed, since the strength of the nonresonant background should not change with respect to the excitation frequency (i.e., the frequency difference between the pump and Stokes pulses). This FM-CARS technique has been demonstrated with narrow-band picosecond lasers by combination of fast frequency modulation of one laser and lockin signal detection. ${ }^{13}$ In beam-scanning microscopy, sophisticated laser systems were required to perform the necessary fast frequency modulation, for example, the one with a novel optical parametric oscillator that can switch the output frequency at tens of megahertz. ${ }^{16}$

Recently, new CARS microscopy methods have emerged based on the so-called "spectral focusing" mechanism. . $^{14,15,17-21}$ These methods can excite a single vibrational level with high spectral resolution by "chirped broad-band" laser pulse pairs (pump and Stokes pulses) and the excitation vibrational frequency can be switched by the time delay between the pulse pair. ${ }^{22}$ Cell and tissue imaging with these methods have already been demonstrated. ${ }^{15,20,23}$ Since the vibrational excitation frequency can be modulated by the time delay (not by the actual frequency of the laser) in this method, the FM technique can be adopted in a relatively simple and low-cost setup. A FM spectral focusing CARS method with passive polarization optics has been demonstrated very recently. ${ }^{14,15}$

In this publication, we introduce a new active version of the FM spectral focusing CARS method that works in the vibrational fingerprint region. Our method allows the use of a laser with a high peak power and lower repetition rate $(\sim 10 \mathrm{~nJ}$ at $2 \mathrm{MHz})$, and we can obtain vibrational images of good quality at the fingerprint region. We show that our FM technique not only removes the nonresonant background but also generates Ramanlike CARS signals. It can perform both background-free CARS imaging and microspectroscopy in real time. We demonstrate its utility with various samples including edible oils, lipid membranes, skin tissues, and plant cell walls.

This paper is organized as follows. In section II, we present the time-domain picture of spectral focusing mechanism and explain fundamental aspects of this method. We also show 
(a)

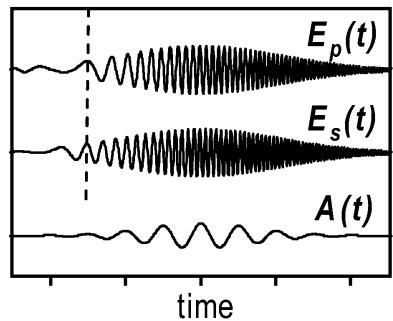

(c)

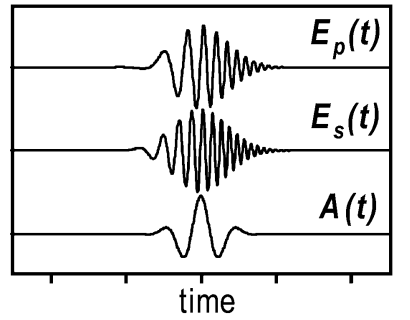

(b)

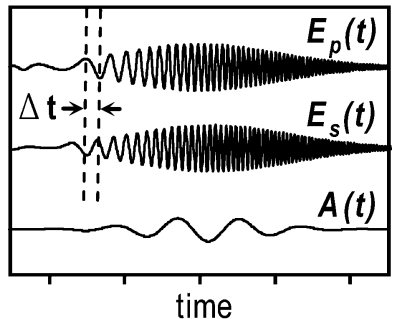

(d)

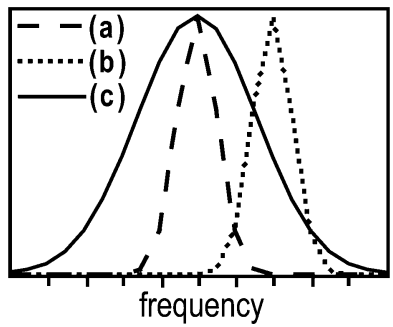

Figure 1. Spectral focusing mechanism. (a and b) Temporal profiles of pump $\left[E_{\mathrm{P}}(t)\right]$, Stokes $\left[E_{\mathrm{S}}(t)\right]$, and excitation $[A(t)]$ fields with a time delay of zero (a) and $\Delta t$ (b), respectively. Note the different modulation periods of $A(t)$ in parts a and b. (c) Temporal profiles with a shorter pulse duration at zero time delay. Note that the modulation period of $A(t)$ is the same as in part a. (d) FT of $A(t)$ from parts a-c. The spectral resolution is higher with longer laser pulses. Also shown is the frequency shift of $A(t)$ by time delay. Traces in parts $\mathrm{a}-\mathrm{c}$ are vertically displaced for clarity. See the text for details.

theoretical comparison of the efficiency of CARS signal generation by the narrow and spectral focusing CARS methods. Section III is the explanation of our experimental method. Section IV begins with the relationship between the amount of chirp and CARS signals studied by phase-controlled laser pulses. FM-CARS spectroscopy and microscopy demonstrations with various samples follow. Section V discusses further important issues, and section VI summarizes the work.

\section{Spectral Focusing CARS}

In most excitation spectroscopy, the spectral resolution is limited by the bandwidth of the excitation light source. Since the natural line width of typical Raman peaks ranges from 5 to $20 \mathrm{~cm}^{-1}$ in the fingerprint region, CARS measurements with femtosecond lasers would have poor spectral resolution. ${ }^{4}$ For example, 100 fs transform-limited (TL) Gaussian pulses at 800 $\mathrm{nm}$ have a bandwidth of $\sim 146 \mathrm{~cm}^{-1}$, which is too broad to resolve individual vibrational peaks. This limit can be circumvented when chirped pulses are used in the CARS process. ${ }^{22}$

Consider the case when broad-band pump and Stokes pulses are stretched by identical group velocity dispersion (GVD, linear chirp). In the time domain, GVD manifests as a linear sweep of the instantaneous laser frequency, as shown in Figure 1a. ${ }^{22}$ Here is shown the case with a positive GVD, where red frequency components arrive earlier than blue ones. Note that the time period of the electric field of the actual laser $(\sim 2.7 \mathrm{fs}$ at $800 \mathrm{~nm}$ ) is much shorter than the pulse durations used in experiments $(\sim 1 \mathrm{ps})$. Thus, we use laser pulses with a much lower carrier frequency for simulations to explain the physical mechanism here. The first step in third-order coherent Raman processes is to excite coherent molecular vibrations by beating frequency between two pulses (pump and Stokes pulses). The frequency of excited coherent vibrations corresponds to the frequency difference between the pump and Stokes pulses. ${ }^{8,22,24}$ Let us consider the nonlinear vibrational excitation field, $A(t)$, by chirped pump and Stokes pulses. Assume that the Stokes pulse is time-delayed with respect to the pump pulse by $\Delta t . A(t)$ is then expressed by $\mathrm{y}^{7,8}$

$$
A(t)=E_{\mathrm{P}}(t) E_{\mathrm{S}}(t, \Delta t)^{*}
$$

where $E_{\mathrm{P}}(t)$ and $E_{\mathrm{S}}(t, \Delta t)$ are the time profiles of the pump and Stokes electric fields, respectively. For linearly chirped pulses under the stationary phase approximation, $E_{\mathrm{P}}(t)$ and $E_{\mathrm{S}}(t, \Delta t)$ can be described by ${ }^{25}$

$$
\begin{gathered}
E_{\mathrm{P}}(t)=E_{\mathrm{P}}(t, \alpha)^{0} \exp \left[\mathrm{i}\left(\omega_{\mathrm{P}}+\frac{t}{2 \alpha}\right) t\right] \\
E_{\mathrm{S}}(t, \Delta t)=E_{\mathrm{S}}(t+\Delta t, \alpha)^{0} \exp \left[\mathrm{i}\left(\omega_{\mathrm{S}}+\frac{(t+\Delta t)}{2 \alpha}\right)(t+\Delta t)\right]
\end{gathered}
$$

where $\omega_{\mathrm{P}}$ and $\omega_{\mathrm{S}}$ are the carrier frequency of the pump and Stokes pulses, respectively. $\alpha$ is GVD, which is equivalent to the coefficient of the quadratic phase modulation in the frequency domain. In this formalism, we separate the time envelope of the electric field $\left[E(t, \alpha)^{0}\right]$ and the frequency-sweeping field component $\{\exp [i(\omega+t / 2 \alpha) t]\}$. Note that the time envelopes, $E_{\mathrm{P}}(t, \alpha)^{0}$ and $E_{\mathrm{S}}(t+\Delta t, \alpha)^{0}$, depend on $\alpha$. The larger $\alpha$ (i.e., higher GVD) is, the longer they become. The quantitative relationship between $\alpha$ and the time profile of electric field envelopes for Gaussian pulses can be found in the previous publications by other workers. ${ }^{19,20} \mathrm{It}$ is straightforward to show that

$$
\begin{aligned}
A(t)=E_{\mathrm{P}}(t, \alpha)^{0} E_{\mathrm{S}}(t+\Delta t, \alpha)^{0} * & \exp (\mathrm{i} \Omega t) \times \\
& \exp \left[-\mathrm{i}\left(\omega_{S}+\frac{\Delta t}{2 \alpha}\right) \Delta t\right]
\end{aligned}
$$

where $\Omega \equiv \omega_{\mathrm{P}}-\omega_{\mathrm{S}}-\Delta t / \alpha$. Note that $\Omega$, the frequency of the excitation field $[A(t)]$, depends on the time delay, $\Delta t$. One can see this phenomenon in the modulation periods of $A(t)$ in Figure 1a,b. It is one of the key advantages of the spectral focusing mechanism; instead of changing the laser frequency, one can switch the vibrational excitation frequency by time delay ${ }^{18,20-22}$ The last term in eq 4 \{i.e., $\left.\exp \left[-\mathrm{i}\left(\omega_{\mathrm{S}}+\Delta t / 2 \alpha\right) \Delta t\right]\right\}$ does not have any effect on measured CARS signals, since it adds an identical phase term to both resonant CARS signals and nonresonant backgrounds. Equation 4 can also explain how the duration of laser pulses affects the spectral resolution of CARS signals. The bandwidth of $A(t)$ is determined by its time duration; the longer $A(t)$ is, the narrower its spectral bandwidth becomes. Figure 1c shows $E_{\mathrm{P}}(t), E_{\mathrm{S}}(t)$, and $A(t)$ from shorter pulse pairs. Since $A(t)$ is shorter in this case, its Fourier transformation has a broader bandwidth, as one can see in Figure 1d. Figure 1d also shows the effective frequency tuning of the excitation field $[A(t)]$ by time delay.

Thus, one can perform high-resolution CARS measurements with chirped broad-band pulses if the time durations of the pump and Stokes pulses are similar to the vibrational dephasing time of molecules (a few picoseconds). ${ }^{18-20}$ Note that this condition depends on both the bandwidth of the laser pulses and the amount of GVD. It also implies that the optimal laser configuration is where the pump and Stokes pulses have the same bandwidths. Remember that the identical GVD of the pump and Stokes pulses are the key requirement of the spectral focusing mechanism (eqs 2-4). If one pulse has a significantly broader bandwidth than the other pulse does, it will be longer in the 
(a)

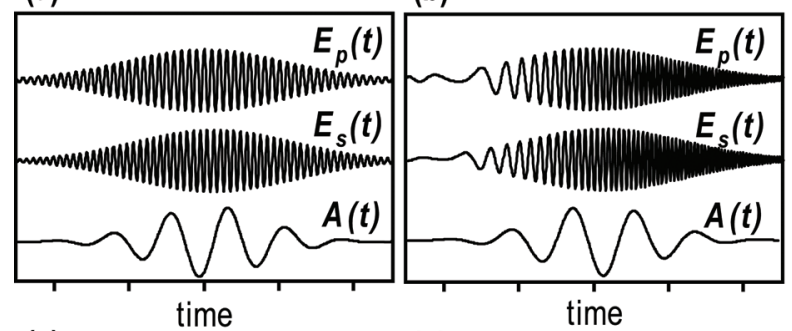

(c)

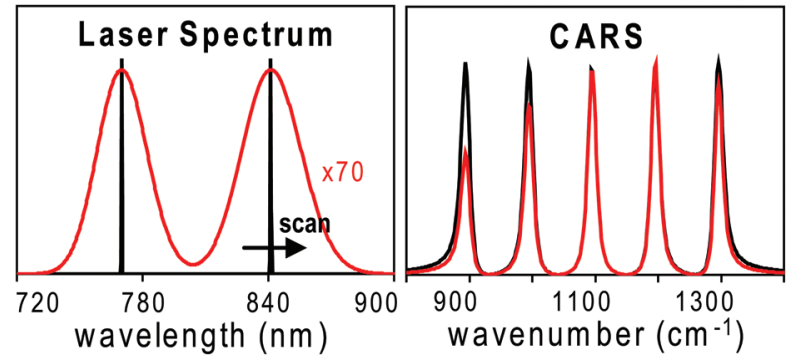

Figure 2. Comparison of CARS methods. (a and b) Temporal profiles of pump $\left[E_{\mathrm{P}}(t)\right]$, Stokes $\left[E_{\mathrm{S}}(t)\right]$ and excitation $[A(t)]$ fields in the narrow (a) and spectral focusing (b) CARS methods, respectively. (c) Laser spectra used in the simulations of part $\mathrm{d}$. The pulse energies are identical in both cases. (d) Simulated CARS spectra of the narrow (black) and spectral focusing (red) methods. Note the similar signal strengths in the center frequency region. Traces in parts $\mathrm{a}$ and $\mathrm{b}$ are vertically displaced for clarity.

time domain (with the same GVD) and some parts of that pulse will not be used for CARS signal generation.

Next, we compare the efficiencies of signal generation by the spectral focusing and narrow CARS methods. Figure 2a,b shows $E_{\mathrm{P}}(t), E_{\mathrm{S}}(t)$, and $A(t)$ in the narrow and spectral focusing methods, respectively. We apply an appropriate GVD to the broad-band pulses in Figure $2 \mathrm{~b}$ such that both cases have identical pulse durations. Note that the phases of $A(t)$ in Figure $2 \mathrm{a}, \mathrm{b}$ are different due to the condition of the simulation, which does not affect the magnitudes of the generated CARS signals. As one can see in Figure 2a,b, amplitudes and shapes of the field envelope of $A(t)$ are identical in both methods. ${ }^{26}$ This can be understood easily by the following consideration. At any given time point, the amplitudes of laser fields in both cases are identical, thus its beating components should be of equal magnitudes.

CARS signals are generated when the pump pulse interacts with the vibrational excitation again. Figure $2 \mathrm{~d}$ shows the simulated CARS signals by each method. The CARS signals is calculated by ${ }^{8}$

$$
\begin{array}{r}
S_{\mathrm{CARS}}(\omega)=\mid \int \mathrm{d} \omega^{\prime} \chi_{\mathrm{R}}\left(\omega^{\prime}\right) E_{\mathrm{P}}\left(\omega-\omega^{\prime}\right) \int \mathrm{d} \omega^{\prime \prime} E_{\mathrm{P}}\left(\omega^{\prime \prime}+\right. \\
\left.\omega^{\prime}\right)\left.E_{\mathrm{S}}\left(\omega^{\prime \prime}\right)\right|^{2}
\end{array}
$$

where $S_{\mathrm{CARS}}(\omega)$ is the intensity of CARS signal photons at the signal frequency of $\omega, \chi_{\mathrm{R}}\left(\omega^{\prime}\right)$ is the third-order vibrational susceptibility of the sample at the vibrational frequency of $\omega^{\prime}$. The laser spectra of the pump and Stokes pulses used in this simulation are shown in Figure 2c. The bandwidths (fwhm) of the narrow and broad CARS pulses are 10 and $450 \mathrm{~cm}^{-1}$, respectively. We apply an appropriate GVD to the broad-band pulses to match the pulse durations to be identical in both cases. The pulse energies $\left(\int|E(\omega)|^{2} \mathrm{~d} \omega\right)$ are also set to be equal. An artificial sample is assumed to possess five vibrational peaks with identical peak intensities. In the case of the narrow CARS method, we scan the frequency of the Stokes pulse to generate a CARS spectrum. In the spectral focusing method, we change the time delay by adding a group delay (i.e., linear spectral phase) to the Stokes pulse. As one can see in Figure 2d, it is clear that both methods have the same signal generation efficiencies as long as the pulse durations are identical. The spectral focusing method generates weaker CARS signals at the lower and higher vibrational frequency regions than the narrow method does. The signals decrease since there are fewer available pairs of the pump and Stokes pulses to excite vibrations at these frequency regions. ${ }^{7-9}$ However, this limitation can be overcome with laser pulses with a broader bandwidth. ${ }^{7}$ In this work, we use laser pulses with a bandwidth of $1800 \mathrm{~cm}^{-1}$ and obtain CARS signals over $800-1700 \mathrm{~cm}^{-1}$ that covers most of the fingerprint region.

In CARS microscopy and spectroscopy, the nonresonant background always accompanies the vibrational CARS signal. ${ }^{1}$ It not only adds up as a smooth background but also interferes with CARS signals to distort its spectral shape. ${ }^{4}$ In CARS imaging at a single vibrational frequency, the nonresonant background can mislead the interpretation of local chemical structures. ${ }^{27,28}$ One method to eliminate the effect of this problematic background is frequency modulation (FM). ${ }^{13-16}$ Since the nonresonant background originates from the electronic response of the sample, its magnitude is insensitive to the vibrational excitation frequency (i.e., $\Omega$ in eq 4). If one can obtain the CARS signals at different $\Omega$ values, the nonresonant background can be subtracted. The FM-CARS technique has been shown to be very effective in eliminating the nonresonant background..$^{13}$ In the previously demonstrated narrow FM-CARS microscopy methods, one needs either two different pump lasers that are switched by a Pockels cell ${ }^{13}$ or a sophisticated optical parametric oscillator (OPO) that can modulate the frequency of the output pulses. ${ }^{16}$ In the spectral focusing CARS, FM can be realized in a much simpler way. Since the excitation frequency can be changed by time delay, FM-CARS can be implemented by switching optical paths, not the actual frequency of laser pulses. ${ }^{14,15}$

\section{Experimental Section}

In this work, we use broad-band pulses from a single cavitydumping Ti:sapphire oscillator laser (Cascade, KM Lasers) to generate CARS signals. The spectrum of our laser is shown in the inset of Figure 3. The output power of this laser is $40 \mathrm{~nJ}$ at a repetition rate of $2 \mathrm{MHz}$. Figure 3 shows our experimental setup for FM-CARS microscopy. The laser beam is split by a 50:50 beam splitter (CVI) and each arm serves as the pump and Stokes pulses. In the pump arm, a Pockels cell (ConOptics, model 350-50C) is used to switch the polarization direction of incoming pulses. Depending on the on-off state of the Pockels cell, the pump pulses travel along one of the two different paths (pump-1 and pump-2 shown in Figure 3) separated by a polarizing beam splitter. These two pump pathlengths are set to be slightly different. The returning pump pulses pass the Pockels cell again to recover the original polarization states. We modulate the Pockels cell with a $100 \mathrm{kHz}$ square driving voltage waveform, which is synchronized to the laser pulse train. In the Stokes arm, we insert a $2.6 \mathrm{~cm}$ long SF57 glass rod (Casix) to match the amount of GVD in the pump and Stokes pulses. The mirror in the Stokes arm is positioned on a computer-controlled translation stage (M.405-CG, PI), which can be delayed at a maximum speed of $1 \mathrm{~mm} / \mathrm{s}$. The pump and 


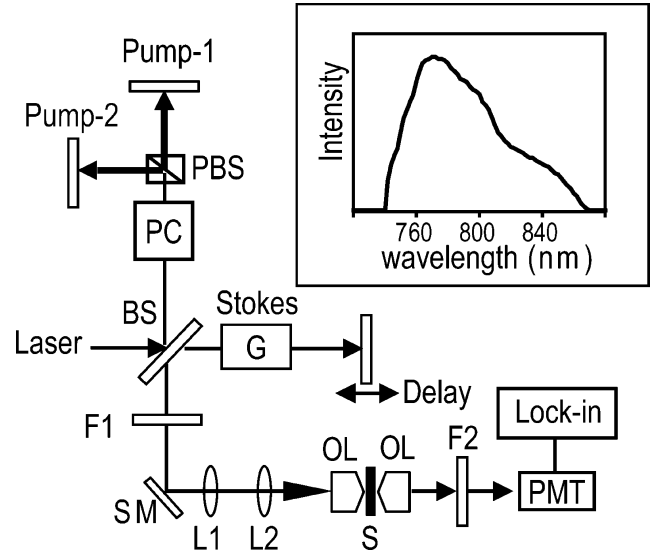

Figure 3. Experimental setup for FM-CARS microscopy. BS, 50:50 beamsplitter; PC, Pockels cell; PBS, polarizing beam splitter; G, SF57 glass; SM, galvo scanning mirror; L1, scan lens; L2, tube lens; OL, microscope objective lens; S, sample; F1, long wavelength pass filter; F2, short wavelength pass filters; Lock-in, lock-in amplifier. (Inset) Laser spectrum.

Stokes pulses are recombined by the beam splitter and travel collinearly afterward. A sharp-edge long wave pass filter (740AELP, Omega Optical) removes laser frequency components shorter than $740 \mathrm{~nm}$. The laser pulses are then sent to a homemade microscope. Our microscope uses a galvo scanner (GVSM002, Thorlabs) to raster scan the laser focal spot on the sample plane. The laser pulses are focused into a sample by a 1.2 NA water immersion objective lens (Olympus) and CARS signals are collected by a 0.65 NA air objective lens (Olympus). The laser pulses are removed by two sharp-edge short wave pass filters (710 ASP, Omega Optical), and CARS signals are measured with a PMT (Hamamatsu, H9656-20). The electrical output of the PMT is fed into a lock-in amplifier (SR830, SRS) referenced to the driving voltage waveform of the Pockels cell. The maximum speed of acquiring an image of $200 \times 200$ pixels is around 1 frame/s ( $25 \mu$ s pixel dwell time). This speed is mostly due to the modulation speed of our Pockels cell (100 $\mathrm{kHz}$ ) and the time constant of the lock-in amplifier. All the spectra shown in this work are taken under the microscope setup in $0.5 \mathrm{~s}$, which is limited by the speed of our current translational stage. Most of CARS measurements in this work are performed with the pump and Stokes powers of 12.0 and $11.0 \mathrm{~mW}$, respectively. These powers are measured before the $1.2 \mathrm{NA}$ objective lens.

The fatty acids used in this work are stearic (18:0), oleic (18: 1), linoleic (18:2), linolenic (18:3), arachidonic (20:4), eicosapentaenoic (EPA) (20:5), and docosahexaenoic (DHA) (22:6) acids. These oils are purchased from Sigma-Aldrich and used without further purification. Oil droplets are prepared in the following way: ${ }^{29} 0.05 \mathrm{~g}$ of each oil is mixed with $0.05 \mathrm{~g}$ of Triton X-100 (Sigma-Aldrich) and stirred for $5 \mathrm{~min}$, and $6 \mathrm{~mL}$ of water is added to the mixture, which is stirred further for $2 \mathrm{~h}$. Separately prepared olive and fish oil droplets are mixed afterward. Multilamellar vesicles (MLV) are prepared from DPPC (1,2-dipalmitoyl-sn-glycero-3-phosphocholine, Avanti Polar Lipids). DPPC powder dissolved in a chloroform $/$ methanol (9:1 vol/vol) solvent is evaporated under vacuum for $6 \mathrm{~h}$ and prehydrated with nitrogen saturated with water vapor for 15 min. A HEPES buffer $(10 \mathrm{mM}, \mathrm{pH} 7.0)$ is then added to the lipid film at $37{ }^{\circ} \mathrm{C}$ and lipid forms MLVs.

For skin imaging, the ear from a white, wild-type mouse is used immediately after sacrificing. A thin sliced skin piece ( $\sim 300 \mu \mathrm{m}$ thick) is immersed in DMSO solvent for $20 \mathrm{~min}$ (a)

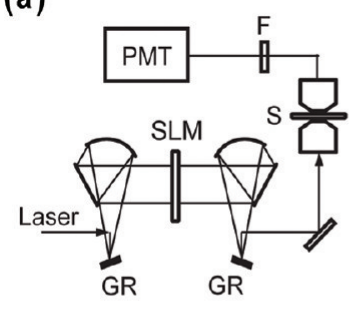

(b)

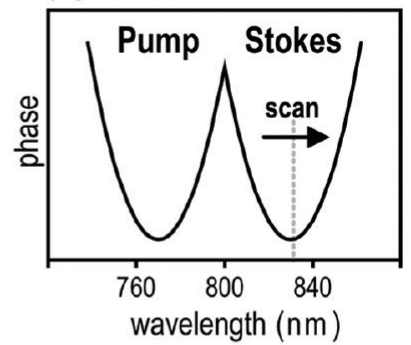

(c)

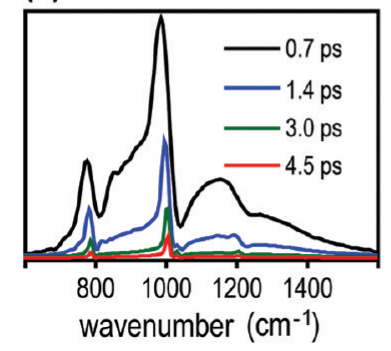

(d)

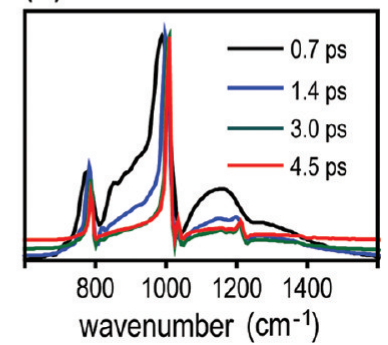

Figure 4. Effect of GVD on spectral focusing CARS. (a) Experimental setup with a pulse shaper. GR, grating; SLM, spatial light modulator; S, sample; F, short wave pass filter. (b) Phase mask for spectral focusing CARS spectroscopy. The center frequency of the Stokes phase mask is shifted to delay the Stokes pulse. (c) Experimentally measured CARS spectra of toluene by pulses with different durations. (d) Normalized traces of part $\mathrm{c}$.

and placed between two coverslips. For plant cell wall imaging, a thin piece of a corn leaf is used.

\section{Results}

Effect of GVD on Spectral Focusing CARS. We first study the effect of the amount of GVD on both spectral resolution and intensity of CARS signals with phase-controlled pulses. The experimental setup is shown in Figure 4a. Note that we use the same laser for both FM-CARS (Figure 3) and the phase-control CARS (Figure 4a) experiments. Detailed explanation of our pulse shaper can be found in our earlier publication. ${ }^{7}$ The microscope, filter, and detector are identical to those used in the FM-CARS setup. The laser pulse is first compressed to be transform-limited at the focus of microscope sample position by the homodyne SPIDER method that we developed recently. ${ }^{30}$ Then, we select the pump and Stokes pulses from a single broadband pulse by applying a phase mask of Figure 4b. The laser frequency region from 740 to $800 \mathrm{~nm}$ is used as the pump and remaining region as the Stokes (Figure 4b). By controlling the slope of the quadratic phase masks (GVD) of Figure $4 b$, we change pulse durations quantitatively. The CARS signal is measured by a PMT while scanning the time delay of the Stokes pulse by shifting the center frequency of its phase mask (Figure $4 b)$. Note that this phase scan corresponds to the time delay of the Stokes pulse. Figure $4 \mathrm{c}$ shows the measured spectral focusing CARS spectra of toluene by pulses of $0.7-4.5$ ps durations. Shorter pulses generate stronger CARS signals. Their vibrational peaks, however, are broad and more distorted (Figure 4c). Figure $4 \mathrm{~d}$ shows the normalized CARS spectra of those in Figure 4c. One can clearly see that the spectral resolution dramatically improves as the pulse is stretched..$^{20}$ However, it cannot exceed the natural line width of the molecular vibration. While the CARS signals from 3 and 4.5 ps pulses show almost identical vibrational linewidths (Figure 4d), the signal strength with 4.5 pulses is only half of the one with 3 ps pulses (Figure 4c).

FM-CARS Spectroscopy. Figure 5a shows spectral focusing CARS spectra of cyclohexane obtained in our FM-CARS setup 
(a)

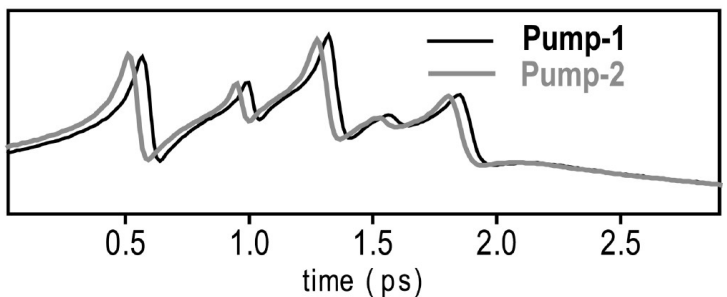

(b)

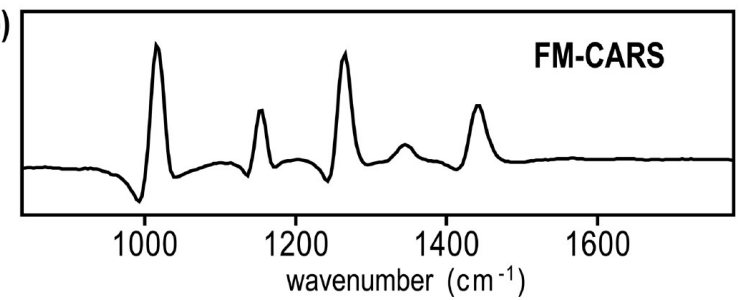

Figure 5. FM-CARS spectroscopy. (a) Measured CARS signals of cyclohexane by the pump-1 (black) and pump-2 (gray) over time delay. The other pump beam is blocked for these measurements. (b) FMCARS signal measured with both the pump-1 and pump-2 pulses. See the text for details.

with only one of the pump-1 and pump- 2 beams. The other pump beam is blocked to obtain these spectra. As emphasized earlier, the key requirement of the spectral focusing CARS method is the identical GVD of the pump and Stokes pulses. ${ }^{18}$ In our experiments, the blue part of the laser pulse acts as the pump and the red part as the Stokes to excite CARS signals at the fingerprint region. We characterize the balance of GVD in the pump and Stokes pulses by the spectral resolution of the CARS spectrum from cyclohexane. We find that the pulse duration of our pump pulse is $1 \mathrm{ps}$ at the sample position without an additional glass in our FM-CARS setup. Note that the Pockels cell, polarizing beam splitter, objective lens, and other optics add a significant GVD on our laser pulses. We experimentally find that inserting an additional $2.6 \mathrm{~cm}$ of a SF57 glass (refractive index of 1.82) in the Stokes arm balances the GVD of pump and Stokes pulses.

As one can see in Figure 5a, the CARS signals are distorted by the nonresonant background, which is well-understood in the CARS community. ${ }^{1}$ With the presence of the nonresonant background, the measured CARS signal is

$$
\begin{array}{r}
S(\omega) \propto\left|P_{\mathrm{R}}(\omega)+P_{\mathrm{NR}}(\omega)\right|^{2}=\left|P_{\mathrm{R}}(\omega)\right|^{2}+P_{\mathrm{NR}}(\omega)^{2}+ \\
2 P_{\mathrm{NR}}(\omega) \operatorname{Re}\left[P_{R}(\omega)\right]
\end{array}
$$

where $S(\omega)$ is the measured signal at the frequency of $\omega$, and $P_{\mathrm{R}}(\omega)$ and $P_{\mathrm{NR}}(\omega)$ are the transient polarizations for the resonant CARS signals and nonresonant backgrounds at $\omega$, respectively. We find that most fingerprint CARS peaks from nonaromatic organic samples fall in the so-called heterodyne limit $\left[P_{\mathrm{R}}(\omega)\right.$ $\left.\ll P_{\mathrm{NR}}(\omega)\right]$ in our method. In this regime, the measured signals become $^{7}$

$$
S(\omega) \propto P_{\mathrm{NR}}(\omega)^{2}+2 P_{\mathrm{NR}}(\omega) \operatorname{Re}\left[P_{\mathrm{R}}(\omega)\right]
$$

Note that the measured signal $[S(\omega)]$ is the CARS signal multiplied by the nonresonant background $\left\{2 P_{\mathrm{NR}}(\omega) \operatorname{Re}\left[P_{\mathrm{R}}(\omega)\right]\right\}$ on top of the nonresonant background $\left[P_{\mathrm{NR}}(\omega)^{2}\right]$. In other words, the CARS signal is amplified by the nonresonant background, which is referred to as heterodyne amplification. ${ }^{13,31}$ In this (a) FM-CARS

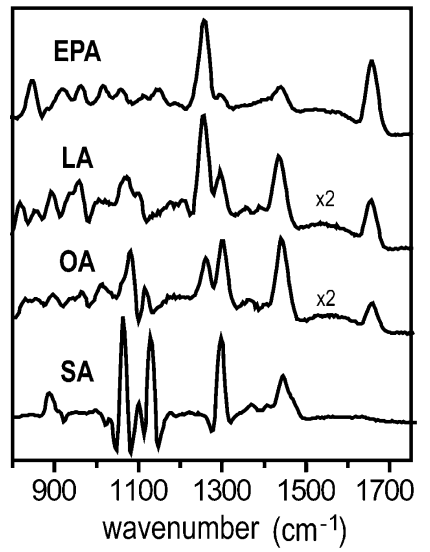

(b) Raman

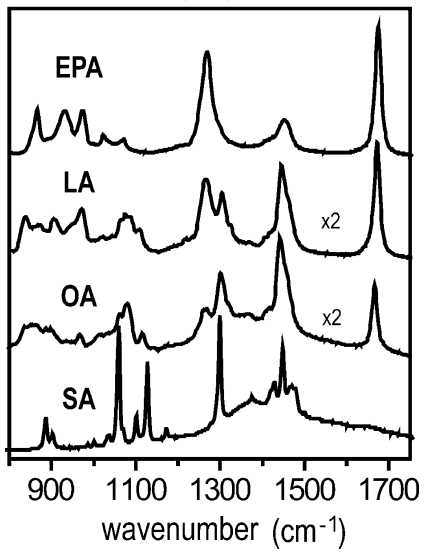

Figure 6. (a) FM-CARS spectra of stearic (SA, 18:0), oleic (OA, 18: 1), and linoleic (LA, 18:2) acids and EPA (20:5). (b) Spontaneous Raman spectra of the same fatty acids. Each trace is vertically displaced for clarity. CARS and Raman spectra for OA and LA are scaled twice.

regime, measured CARS signals also become linearly proportional to sample concentration. ${ }^{13,29}$

When both pump pulses are unblocked, the lock-in amplifier measures the difference between these two CARS signals, and the spectral shape of its output looks close to the shape of spontaneous Raman scattering (Figure 5b). Since we can monitor the entire CARS spectrum in real time by fast scanning of the Stokes pulse delay, we adjust the mirror position of the pump-2 to maximize the spectral resolution of FM-CARS signals with minimum loss of its peak intensity. ${ }^{14}$ The entire vibrational spectrum over $800-1700 \mathrm{~cm}^{-1}$ is measured by scanning the time delay of the Stokes pulses. Despite of the existence of small dips around the peaks, the FM-CARS spectrum reveals narrow individual vibrational peaks and can be directly correlated to the spontaneous Raman spectrum. We convert time delay into vibrational frequency with the known Raman peak positions of cyclohexane. An excellent linear correlation between the time delay and vibrational frequency is found. Due to the mismatched high-order chirps between the pump and Stokes pulses, we cannot maximize the spectral resolution over the entire spectral range. One can notice that the $1444 \mathrm{~cm}^{-1}$ peak of cyclohexane in Figure $5 \mathrm{~b}$ is slightly broader than other peaks. As one can see in Figure 5b, however, we are able to achieve good spectral resolution over $900-1500 \mathrm{~cm}^{-1}$. The fwhm of the $1028 \mathrm{~cm}^{-1}$ peak in Figure $5 \mathrm{~b}$ is around $20 \mathrm{~cm}^{-1}$.

Quantitative Chemical Identification of Fatty Acids. Figure 6 shows the FM-CARS and spontaneous Raman spectra of stearic (18:0), oleic (18:1), and linoleic (18:2) acids and EPA (20:5). Since these spectra contain rich information from multiple characteristic vibration peaks such as olefinic $\mathrm{C}=\mathrm{C}$ and methylene $\mathrm{CH}_{2}$ deformations, it is possible to identify molecular structures by quantitative spectral analysis. ${ }^{32,33}$ Raman spectra in Figure $6 \mathrm{~b}$ are measured under the same microscope with a homemade confocal Raman microscope that consists of a $785 \mathrm{~nm}$ continuous wave laser and a spectrometer (Holospec f/1.8, Kaiser Optical System) coupled with a cooled CCD (iDus DU420A, Andor). We observe all the major Raman peaks in the FM-CARS spectra. Vibrational peak positions of FM-CARS spectra are matched with those of Raman spectra within 10 $\mathrm{cm}^{-1}$. The relative peak intensities in the FM-CARS and spontaneous Raman spectra, however, show some degree of differences. This is mainly due to the limited bandwidth of our laser pulses, as explained in section II. ${ }^{7}$ We also notice that the peak ratio in FM-CARS is significantly different from that in 

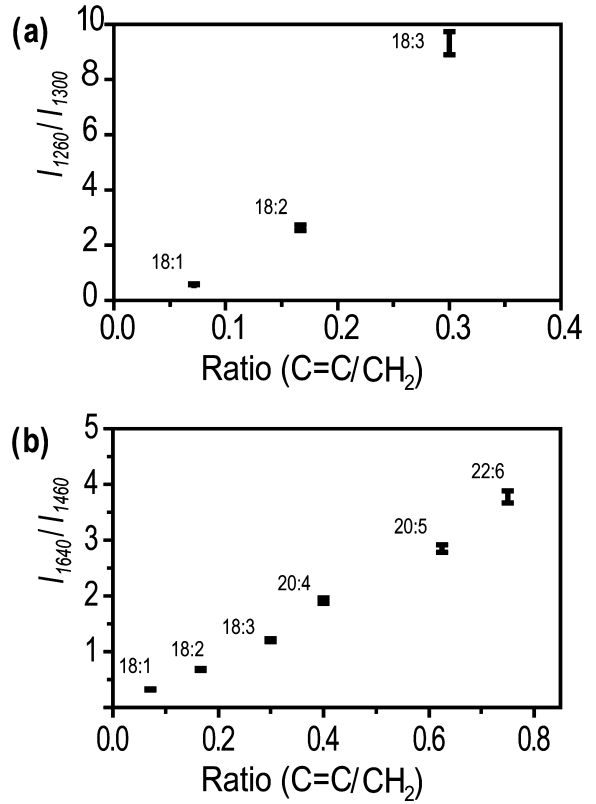

Figure 7. The intensity ratios (a) $I_{1260} / I_{1300}$ and (b) $I_{1640} / I_{1460}$ versus the unsaturation level of fatty acids. The unsaturation level is calculated by the number of $\mathrm{C}=\mathrm{C}$ bonds per $\mathrm{CH}_{2}$. In the plot also shown are the conventional notations $(X: Y)$ for fatty acids. The vertical error bars represent the standard deviations over 10 independent measurements.

Raman when the peaks are very close to each other. One example is the 1260 and $1300 \mathrm{~cm}^{-1}$ peaks in Figure 6a. We emphasize, however, that the relative peak ratio in FM-CARS spectra of the same sample is consistent from different measurements. Thus, we can identify the sample by the vibrational peak analysis once we know the FM-CARS spectrum of the pure sample.

Figure 7 shows examples of quantitative CARS spectral analysis. Here, we measure the FM-CARS spectra of a series of fatty acids that have different unsaturation levels. Note that the notation $X: Y$ here corresponds to the numbers of carbon atoms $(X)$ and double bonds $(Y)$ in the acyl chain, respectively. In the fingerprint region, there are several distinct features in Raman spectra of fatty acids (Figure 6b). In the following discussion, we round the vibrational peak positions to the nearest tens.

C-C Stretching Region $\left(1000-1200 \mathrm{~cm}^{-1}\right)$. This region contains peaks due to the gauche and trans conformations of the acyl chains. ${ }^{32,34,35}$ Liquid fatty acids show weak featureless bands while solid ones have distinct triplet peaks. This feature is a great indicator of the thermodynamic phase of fatty acids and phospholipids.

Intensity Ratio of 1260 and $1300 \mathrm{~cm}^{-1}$ Peaks. In the literature, these peaks are assigned to in-plane olefinic hydrogen bending $\left(1260 \mathrm{~cm}^{-1}\right)$ and methylene twisting deformation (1300 $\left.\mathrm{cm}^{-1}\right){ }^{32,34}$ The 1260 and $1300 \mathrm{~cm}^{-1}$ peak intensities increase with the numbers of $\mathrm{C}=\mathrm{C}$ double bonds and $\mathrm{C}-\mathrm{C}$ single bonds, respectively. ${ }^{36}$ Note that the relative intensity of $1260 \mathrm{~cm}^{-1}$ peak per a $C=C$ double bond is significantly higher than that of 1300 $\mathrm{cm}^{-1}$ peak per a $\mathrm{C}-\mathrm{C}$ single bond. Thus, the $1260 \mathrm{~cm}^{-1}$ peak dominates over the $1300 \mathrm{~cm}^{-1}$ one in highly unsaturated fatty acids such as EPA (20:5). Figure 7a shows the peak ratios of 1260 and $1300 \mathrm{~cm}^{-1}\left(I_{1260} / I_{1300}\right)$ of oleic $(18: 1)$, linoleic $(18: 2)$, and linolenic (18:3) acids. We plot $I_{1260} / I_{1300}$ against the ratio of the number of $\mathrm{C}=\mathrm{C}$ double bonds over the number of methylene $\left(-\mathrm{CH}_{2}-\right)$ carbon atoms in the acyl chain [referred to ratio $\left(\mathrm{C}=\mathrm{C} / \mathrm{CH}_{2}\right)$ in the horizontal axis of Figure 7]. ${ }^{32}$ As one can see in Figure 7a, we find a good correlation of this
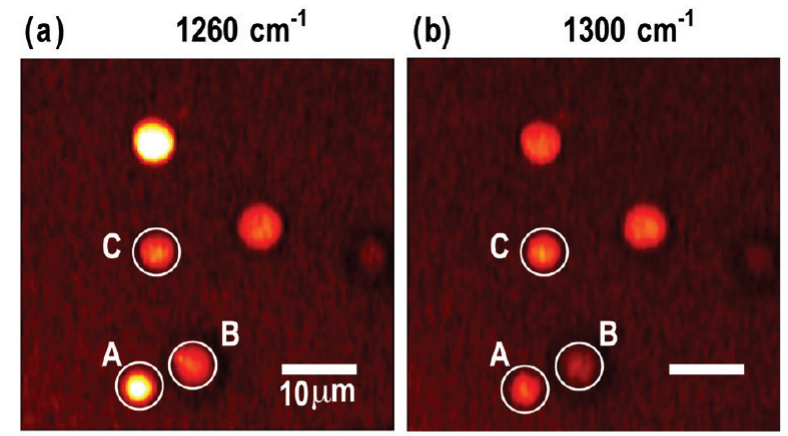

(c) $\quad I_{1260} I I_{1300}$

(d)
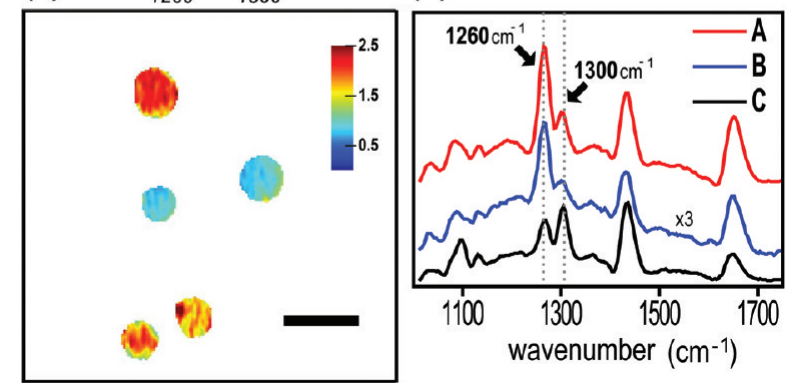

Figure 8. FM-CARS images at (a) $1260 \mathrm{~cm}^{-1}$ and (b) $1300 \mathrm{~cm}^{-1}$ of a mixture of fish and olive oil droplets. The images have $200 \times 200$ pixels and the pixel size is $250 \times 250 \mathrm{~nm}^{2}$. The acquisition time for each image is $1 \mathrm{~s}$. (c) Intensity ratio $\left(I_{1260} / I_{1300}\right)$ image of $1260 \mathrm{~cm}^{-1}$ over $1300 \mathrm{~cm}^{-1}$ peaks. The only area with significant intensity at 1260 $\mathrm{cm}^{-1}$ is shown with a false color code. Olive and fish oil droplets appear blue and reddish yellow, respectively. The scale bar is $10 \mu \mathrm{m}$. (d) FMCARS spectra of the oil droplets marked as A, B, and C in parts a and b. The trace of B is scaled by 3 times. All the traces in part d are vertically displaced for clarity.

peak ratio $\left(I_{1260} / I_{1300}\right)$ and the unsaturation level of fatty acids. ${ }^{36}$ This parameter $\left(I_{1260} / I_{1300}\right)$ is a good indicator of the relative number of $\mathrm{C}=\mathrm{C}$ double bonds in fatty acids and phospholipids that contain one to three $\mathrm{C}=\mathrm{C}$ double bonds per acyl chain.

1460 and $1640 \mathrm{~cm}^{-1}$ Peaks. These peaks are assigned to methylene scissor deformation $\left(1460 \mathrm{~cm}^{-1}\right)$ and $\mathrm{C}=\mathrm{C}$ double bond stretching $\left(1640 \mathrm{~cm}^{-1}\right)$, respectively. ${ }^{32-34}$ The $1460 \mathrm{~cm}^{-1}$ peak is present in the spectra of all fatty acids. ${ }^{33}$ The intensity ratio of 1460 over $1640 \mathrm{~cm}^{-1}$ peaks serves as another indicator of lipid unsaturation level over broader sample ranges. Figure $7 \mathrm{~b}$ shows the peak ratio of 1640 over $1460 \mathrm{~cm}^{-1}\left(I_{1640} / I_{1460}\right)$ versus the $\mathrm{C}=\mathrm{C} / \mathrm{CH}_{2}$ ratio. It shows a good pseudolinear relationship.

These features provide rich chemical information about acyl chains of fatty acids and lipids. They serve as means of chemical selectivity in oil droplet and phospholipid membrane imaging in the next sections.

Chemical Imaging of Oil Droplets. Figure 8a,b shows the FM-CARS images of a mixture of olive and fish oil droplets at 1260 and $1300 \mathrm{~cm}^{-1}$, respectively. ${ }^{29}$ The main difference between their chemical structures is the degree of unsaturation in their acyl chains. Olive oil consists of oleic acid $(18: 1)$ primarily $\left(72 \%{ }^{37}\right)$, while fish oil contains a large amount $\left(50 \%{ }^{37}\right)$ of polyunsaturated fatty acids such as DHA (22:6) and EPA (20:5). Note that both oils possess vibrational peaks at 1260 and $1300 \mathrm{~cm}^{-1}$. Although one can see larger signals from fish oil droplets than the olive ones at $1260 \mathrm{~cm}^{-1}$, it is qualitative at best and may lead to false chemical identification. For example, the droplet B in Figure 8a is a fish oil droplet positioned vertically off from the laser focus. Its apparent signal strength at $1260 \mathrm{~cm}^{-1}$ is similar to that of the olive oil droplet with a 
(a)
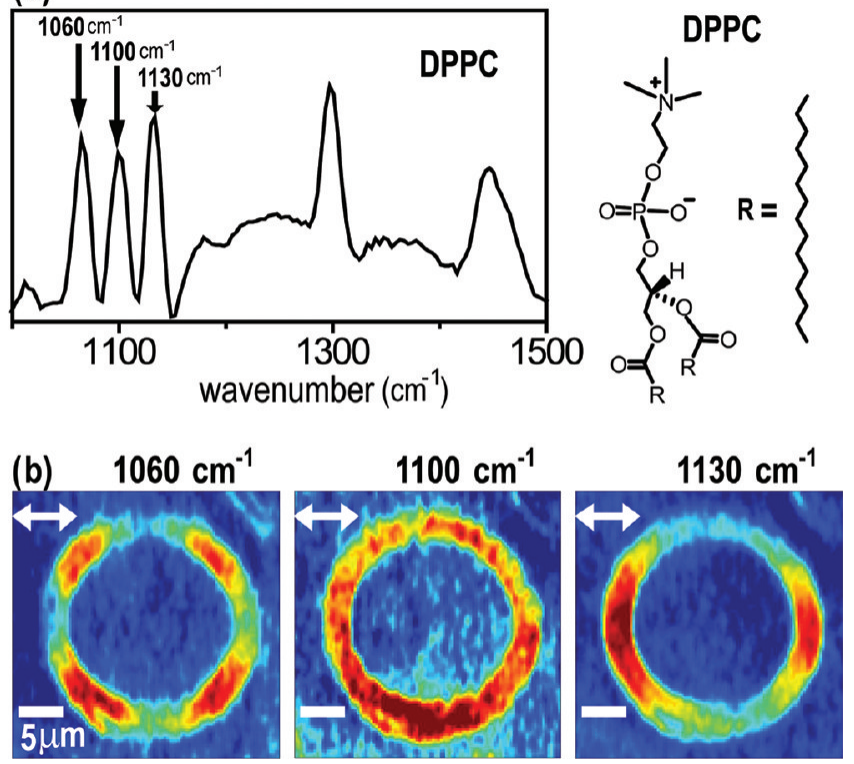

Figure 9. (a) FM-CARS spectrum of bulk DPPC film. The molecular structure of DPPC is also shown. (b) FM-CARS images of a DPPC MLV at 1060,1100, and $1130 \mathrm{~cm}^{-1}$. Each image has $100 \times 100$ pixels and the pixel size is $300 \times 300 \mathrm{~nm}^{2}$. The acquisition time for each image is $10 \mathrm{~s}$. The polarization directions of the laser pulses are indicated with white arrows. Note that the intensity scale of each image is scaled for clarity. The image at $1130 \mathrm{~cm}^{-1}$ has about 3 times stronger signals than the one at $1100 \mathrm{~cm}^{-1}$. The scale bar is $5 \mu \mathrm{m}$. See the text for details.

similar size (C in Figure 8a). However, when we compare the CARS images at $1260 \mathrm{~cm}^{-1}$ (Figure 8a) and $1300 \mathrm{~cm}^{-1}$ (Figure $8 b$ ), it is obvious that this droplet is fish oil. We can quantitatively identify these droplets by taking the ratio image as shown in Figure 8c. It clearly distinguishes these two different oils. We find that the intensity ratios of 1260 and $1300 \mathrm{~cm}^{-1}$ peaks $\left(I_{1260} / I_{1300}\right)$ are $\sim 2.5$ and $\sim 0.8$ for fish and olive oils, respectively. We can also instantly switch our experiment to the microspectroscopy mode. Figure 8d shows three FM-CARS spectra taken at the droplets A, B, and C. We position the laser focus at the point of interest and scan the time delay to obtain these spectra. Thus, we are able to not only acquire chemical images at several important frequencies in real-time but also perform microspectroscopy at a few positions of interest. Combination of multiple frequency imaging and microspectroscopy will be a powerful tool in the study of complex samples such as cells and tissues.

Polarization Dependent Imaging of DPPC Multilamellar Vesicle. We investigate the orientational order of lipid acyl chains in phospholipid membranes by polarization dependence of CARS signals. Multilamellar vesicles (MLV) of DPPC (its chemical structure is shown at the upper right corner in Figure 9) are used in this study. DPPC has two completely saturated alkyl chains, and its membrane is in a gel phase at room temperature. ${ }^{34}$ Figure 9a shows the FM-CARS spectrum of a thick bulk DPPC film. Note that the CARS spectrum of DPPC membrane can be significantly different from this bulk spectrum. DPPC has distinct triplet peaks around $1100 \mathrm{~cm}^{-1}$ in solid or gel phases. In the literature, the 1060 and $1130 \mathrm{~cm}^{-1}$ peaks are assigned to out-of-phase and in-phase $\mathrm{C}-\mathrm{C}$ stretching vibrations of all-trans conformers, respectively. ${ }^{32,34}$ The narrow $1100 \mathrm{~cm}^{-1}$ peak is the $\mathrm{C}-\mathrm{C}$ stretching mode of conformers with isolated gauche defects. ${ }^{34,35}$

Interestingly, we see distinct polarization dependence of CARS images in this frequency region. Figure $9 \mathrm{~b}$ shows the
CARS mages at 1060,1100 , and $1130 \mathrm{~cm}^{-1}$. Note that all the laser pulses in our FM-CARS method have identical polarization directions. This is an advantage of our method over the previously demonstrated FM-CARS methods, which utilized two orthogonally polarized pulses. ${ }^{14,15}$ The white arrow in Figure $9 \mathrm{~b}$ indicates the polarization direction of our laser pulses. The CARS images at 1060 and $1130 \mathrm{~cm}^{-1}$ are highly polarized (Figure 9b) and show the shapes of a quadrupole and dipole, respectively. These vibrational peaks are due to $\mathrm{C}-\mathrm{C}$ stretching of all-trans acyl chains, which form ordered zigzag chain conformations confined in a 2D plane. The image at $1100 \mathrm{~cm}^{-1}$, the gauche conformer peak, is almost depolarized. Since gauche conformers represent disordered chain conformation, it is expected to be depolarized. Note that we scale the image intensities to visualize the contrast of each image in Figure 9b. The signal intensity at $1100 \mathrm{~cm}^{-1}$ is significantly lower than those of 1060 and $1130 \mathrm{~cm}^{-1}$, implying that this membrane is highly ordered but still possesses a small amount of gauche defects. As one can see in this study, polarization-dependent CARS imaging can give valuable information for the chain order of lipids. Further investigation is currently under progress.

Imaging of Chemical Diffusion in Skin. CARS microscopy is an excellent tool to track chemicals in tissues if the chemical has a distinct Raman peak that is not present in the tissue. ${ }^{1,2}$ Figure 10 shows such an example. Here we study the penetration of dimethyl sulfoxide (DMSO) in mouse skin tissue. DMSO is widely used as a skin-penetration enhancer and diffuses through hydrophilic parts of skin tissues. ${ }^{38}$ Figure 10a,b shows the distribution of DMSO and fat in the subcutaneous fat layer at a depth of $65 \mu \mathrm{m}$ from the skin surface. These images are obtained at 1027 and $1460 \mathrm{~cm}^{-1}$ for DMSO and fat, respectively. One can clearly see that DMSO penetrates the skin via hydrophilic regions and avoids lipid-concentrated area. The depth profiles in Figure 10c,d show that our imaging depth is up to $80 \mu \mathrm{m}$. We observe several lipid-rich structures in the skin. In Figure 10d, bright area corresponds to stratum corneum, sebaceous glands, and subcutaneous fat layers from bottom (skin surface) to top.

FM-CARS spectra of DMSO and fat cells are shown in Figure 10e. DMSO has a peak at $1420 \mathrm{~cm}^{-1}$ close to our probe frequency of fats $\left(1460 \mathrm{~cm}^{-1}\right)$. The image at $1460 \mathrm{~cm}^{-1}$, however, shows a clear distribution of fat, confirming the high spectral resolution of our FM-CARS method. When we switch the excitation frequency from 1460 to $1420 \mathrm{~cm}^{-1}$ and the corresponding image shows the distribution of DMSO (data not shown).

Chemical Imaging of Plant Tissues. Figure 11 shows the FM-CARS images of plant cell walls in a corn leaf. This sample represents an example of lignocellulosic biomass that gains significant interest recently. ${ }^{1,39}$ Since cellulose can be processed into alcohol, it is considered as an alternative renewable energy source. Lignin is one of the major obstacles in this process, since it prevents decomposition of cellulose polymers into soluble saccharides. ${ }^{1,39}$ Thus, understanding the microscopic structure of lignin and cellulose in plant tissues becomes an important technological question..$^{39}$ We image both cellulose and lignin at 1100 and $1600 \mathrm{~cm}^{-1}$, respectively. The $1100 \mathrm{~cm}^{-1}$ peak is from the stretching vibrations of the $\mathrm{C}-\mathrm{O}-\mathrm{C}$ linkage of cellulose ${ }^{40}$ Lignin's $1600 \mathrm{~cm}^{-1}$ peak originates from its aromatic $\mathrm{C}=\mathrm{C}$ vibrations and is used as a lignin marker in previous Raman and CARS microscopy studies. ${ }^{1,39,40}$ As one can see in Figure 11a,b, these two components have distinct spatial distributions. The vertical sections of parts $\mathrm{a}$ and $\mathrm{b}$ of Figure 


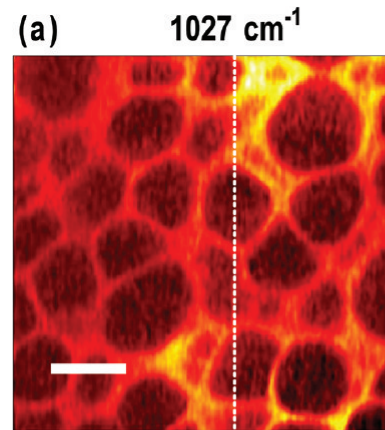

(b) $\quad 1460 \mathrm{~cm}^{-1}$

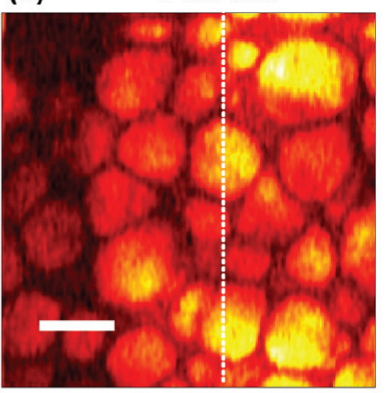

(c)

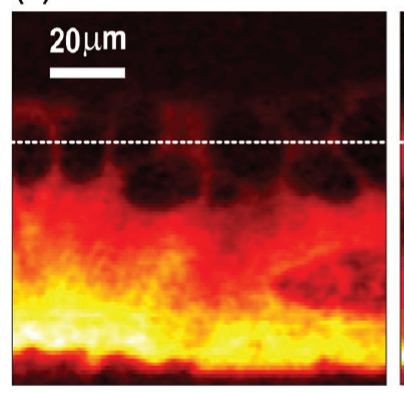

(d)

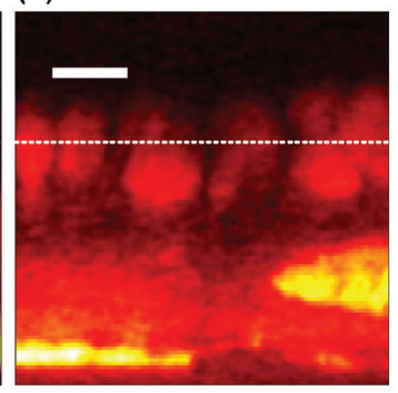

(e)

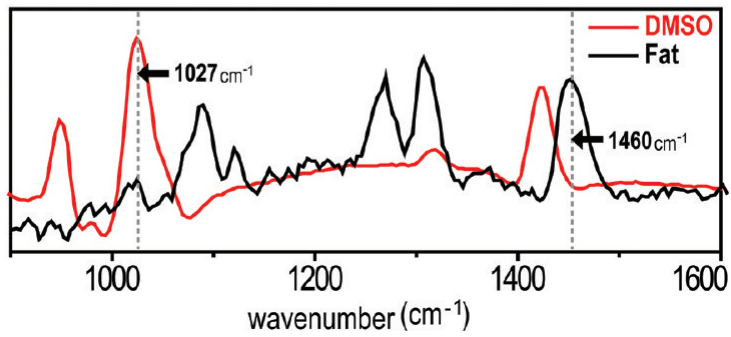

Figure 10. FM-CARS images of (a) DMSO $\left(1027 \mathrm{~cm}^{-1}\right)$ and (b) fat $\left(1460 \mathrm{~cm}^{-1}\right)$ in a skin tissue previously soaked in DMSO. The depth profiles of (c) DMSO and (d) fat are along the white line indicated in parts $a$ and $b$. The vertical section position of images in parts $a$ and $b$ are also indicated by the white line in parts $\mathrm{c}$ and $\mathrm{d}$. The lateral images ( $\mathrm{a}$ and $\mathrm{b}$ ) have $200 \times 200$ pixels and the pixel size is $500 \times 500 \mathrm{~nm}^{2}$. The depth profiles are constructed from 100 stacks of lateral images. It takes $7 \mathrm{~s}$ to obtain one lateral image. The scale bar is $20 \mu \mathrm{m}$. (e) FM-CARS spectra of DMSO and fat.

11 are also shown in parts $\mathrm{c}$ and d, respectively. One can clearly see that lignin layers are sandwiched between cellulose layers.

We also perform microspectroscopy of this sample. We find that the photodamage threshold of this sample is rather low, and $0.5 \mathrm{~s}$ laser exposure at a single spatial point is only possible with a laser power less than $0.5 \mathrm{~mW}$. However, fast beam scanning microscopy can be performed with a laser power up to $20 \mathrm{~mW}$ without any visible photodamage. Thus, instead of measuring the FM-CARS spectrum at a single position, we obtain 80 images at vibrational frequencies from 900 to 1700 $\mathrm{cm}^{-1}$ at $10 \mathrm{~cm}^{-1}$ intervals. Each frequency image is taken in $1.6 \mathrm{~s}$, resulting in a total hyperspectral imaging time of $2 \mathrm{~min}$. In Figure 11e, we show the vibrational spectra at two sample positions (marked as A and B in Figure 11a,b) constructed by this way.

Sensitivity Limit of the Current FM-CARS Method. We test the sensitivity of our FM-CARS with aqueous $\mathrm{NaNO}_{3}$ solutions of different concentrations. The measured concentration dependence of the $1050 \mathrm{~cm}^{-1}$ peak of $\mathrm{NO}_{3}{ }^{-}$ions is shown in Figure 12a. In this measurement, the powers of the pump and Stokes pulses before the focusing microscope objective are 10.0 and $8 \mathrm{~mW}$, respectively. The lock-in time constant of 1 $\mathrm{ms}$ is used in this measurement. As one can see in Figure 12a,
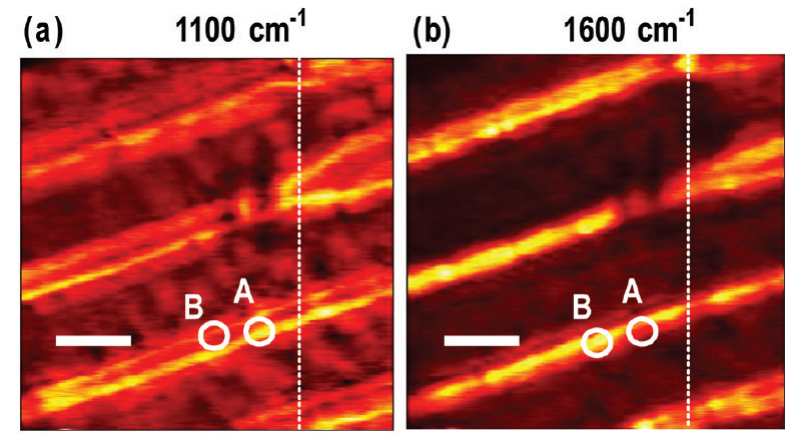

(c)

(d)
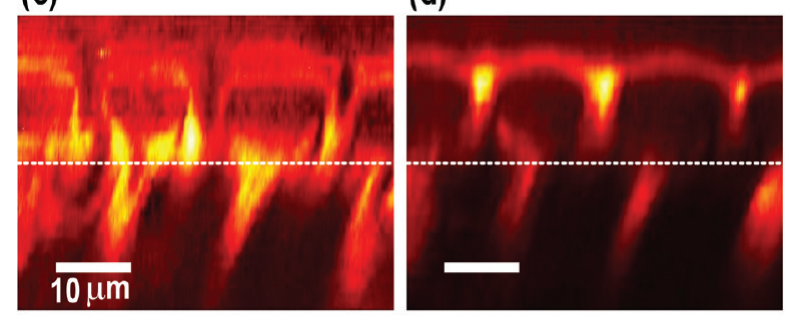

(e)

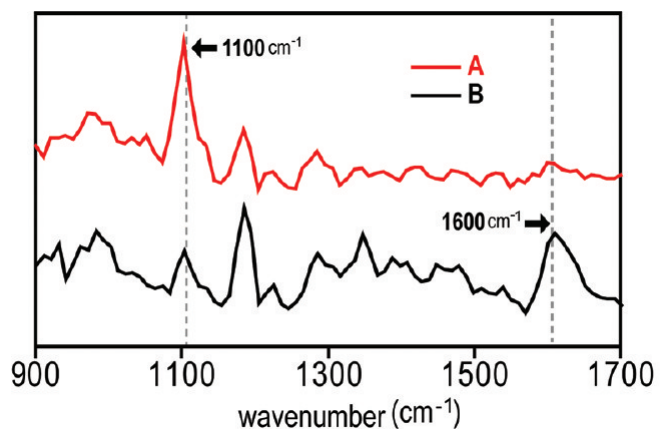

Figure 11. FM-CARS images of (a) cellulose $\left(1100 \mathrm{~cm}^{-1}\right)$ and (b) lignin $\left(1600 \mathrm{~cm}^{-1}\right)$ in a corn leaf. The depth profiles of (c) cellulose and (d) lignin are along the white line indicated in parts a and b. Parts $\mathrm{a}$ and $\mathrm{b}$ are measured at the vertical position indicated by the white line in $\mathrm{c}$ and $\mathrm{d}$. The lateral images $(\mathrm{a}$ and $\mathrm{b})$ have $300 \times 300$ pixels. It takes $1.6 \mathrm{~s}$ to obtain one lateral image. The depth profiles are constructed from 40 stacks of lateral images. The scale bar is $10 \mu \mathrm{m}$. (e) FMCARS spectra at the positions marked as A and B in parts a and b.
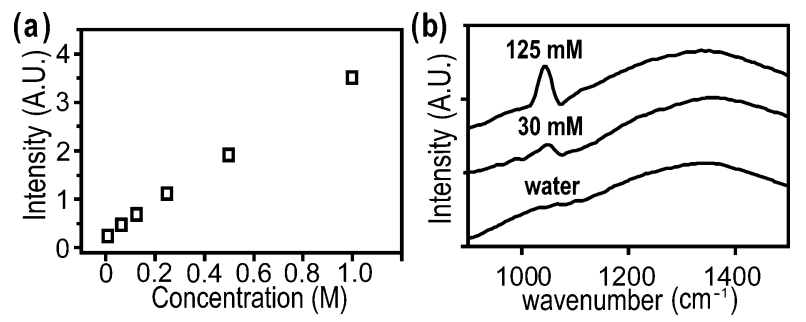

Figure 12. (a) Concentration dependence of FM-CARS signals from aqueous $\mathrm{NaNO}_{3}$ solutions. (b) FM-CARS spectra of $\mathrm{NaNO}_{3}$ solutions at two representative low concentrations. Spectra of water is also shown for comparison. Each trace in part b is vertically displaced for clarity.

a linear concentration dependence of FM-CARS signal is obvious. This is due to the fact that our FM-CARS measures the cross term between the vibrational CARS signal and nonresonant background $\left\{2 P_{\mathrm{NR}}(\omega) \operatorname{Re}\left[P_{\mathrm{R}}(\omega)\right]\right.$ in eqs 6 and 7$\}$. Thus, the signal should be linearly proportional to the sample concentration if the nonresonant background $\left[P_{\mathrm{NR}}(\omega)\right]$ is invariant. ${ }^{13}$ Figure $12 \mathrm{~b}$ shows FM-CARS spectra of $\mathrm{NaNO}_{3}$ solutions at the concentrations of 125 and $30 \mathrm{mM}$. The FM-CARS spectrum of pure water is also shown for comparison. From this measurement, we estimate the current detection limit to be $\sim 10 \mathrm{mM}$ of $\mathrm{NO}_{3}{ }^{-}$ions with a $1 \mathrm{~ms}$ lock-in time constant. 
In Figure 12b, one can see a smooth background centered around $1400 \mathrm{~cm}^{-1}$. This is due to the slightly different magnitudes of nonresonant backgrounds between the CARS signals generated by the pump- 1 and -2 . The cross-correlation time duration of CARS signals from a glass coverslip (nonresonant sample) is $\sim 1.5 \mathrm{ps}$ (fwhm). Meanwhile, the time delay between the pump- 1 and -2 is set to 50 fs in this work. Accordingly, there is residual nonresonant background, which can be as large as $\sim 3 \%$ of its original strength in our experiment. Note that this residual nonresonant background is not significant in the samples with strong resonant CARS signals but becomes pronounced for samples with weak signals. This residual nonresonant background limits our detection sensitivity and vibrational selectivity. We find that this residual nonresonant background fluctuates during microspectroscopy measurements of small samples, probably due to the laser trapping action of our focused laser pulses. This also limits our sensitivity of microspectroscopy.

\section{Discussion}

It is important to consider the optimal laser configuration for spectral focusing CARS microscopy. As mentioned in the section II, the critical requirement of this method is the identical GVD in the pump and Stokes pulses. In addition, the duration of time overlap between the two pulses determines the spectral resolution of the CARS signal. Thus, the optimal condition is met when the bandwidths of the pump and Stokes pulses are identical. ${ }^{26}$ Note that the strength of a pure resonant CARS signal at its peak frequency does not depend significantly on the pulse duration if the pulses are shorter than the vibrational dephasing time. ${ }^{8,26}$ However, as we demonstrated experimentally in Figure 4, CARS signals increase with shorter laser pulses (i.e., less GVDs) by the heterodyne amplification of the CARS signal with the nonresonant background as a local oscillator. ${ }^{13,31}$ The nonresonant background is an electronic response and has a virtually instantaneous response time. A shorter pulse will boost the strength of this background and increase its interference with the CARS signal $\left\{2 P_{\mathrm{NR}}(\omega) \operatorname{Re}\left[P_{\mathrm{R}}(\omega)\right]\right.$ in eqs 6 and 7$\} .{ }^{4}$ Accordingly, one can obtain stronger signals with shorter pulses. The spectral resolution, however, becomes poor if the pulse is too short. We find that the optimum balance between CARS signal strength and the spectral resolution occurs at $\sim 1$ ps pulse duration. This is the condition of our experiments reported here. The cross-correlation of the pump and Stokes pulses measured from the nonresonant background with a glass is $1.5 \mathrm{ps}$.

Another important issue that one must consider in multiphoton microscopy is nonlinear laser photodamage of samples. In our previous works, we used very short laser pulses to generate CARS signals. ${ }^{7,29} \mathrm{We}$ observe that $30 \mathrm{fs}$ pulses with a power of $2 \mathrm{~mW}(1 \mathrm{~nJ}$ in $2 \mathrm{MHz}$ ) focused by a $1.2 \mathrm{NA}$ objective can instantly blast cells and tissues. However, 1 ps pulses with as much power as $20 \mathrm{~mW}(10 \mathrm{~nJ}$ in $2 \mathrm{MHz})$ can be tolerated by live cells without any visible short-term photodamage. Since the CARS process is a third-order process, one can obtain orders of magnitude larger CARS signals with picosecond pulses than with femtosecond pulses under the limit of sample photodamage. Note that photodamage is a complicated phenomenon that depends on the wavelength of the laser, the laser repetition rate, and the samples. Our observations also agree with the result from the previous photodamage study of living cells and tissues. ${ }^{41}$ We also notice that the photodamage threshold critically depends on the laser dwell time. As discussed in the lingnin/cellulose imaging (Figure 11), fast beam-scanning microscopy can be performed with much higher laser powers than point microspectroscopy measurements in several cases. In this situation, hyperspectral imaging by fast beam scanning microscopy will be more appropriate.

Most biomedical CARS imaging has been performed at the $2840 \mathrm{~cm}^{-1}$ peak. ${ }^{1}$ This symmetric $\mathrm{CH}_{2}$ stretching peak is uniquely isolated from other peaks and very strong in lipid samples due to its abundance in lipid acyl chains. Since the CARS cross section is quadratically proportional to that of spontaneous Raman scattering, it implies that the $2840 \mathrm{~cm}^{-1}$ peak is significantly stronger than most peaks in the fingerprint region. However, as we demonstrate in this work, the fingerprint region can offer rich chemical information.

Another noteworthy difference of vibrational response of biological samples at the fingerprint and $\mathrm{CH}$-stretching regions are their spectral shapes. As demonstrated in this work, one can often find narrow resolved vibrational peaks in the fingerprint region. In addition, CARS signals from most biological samples fall in the heterodyne regime $\left(P_{\mathrm{R}} \ll P_{\mathrm{NR}}\right)$. Under this condition, spectral shapes of measured CARS signals are dispersive and our FM method can be applied to generate Raman-like CARS signals. In the $\mathrm{CH}$ stretching region $\left(2800-3000 \mathrm{~cm}^{-1}\right)$, however, dense lipid structures such as lipid droplets fall in the homodyne regime $\left(P_{\mathrm{R}} \gg P_{\mathrm{NR}}\right)$ and the spectral shapes of CARS signals are often very close to that of spontaneous Raman scattering. ${ }^{20}$ The peaks in this region are also very crowded and one cannot distinguish individual peaks. ${ }^{42}$ Our FM-CARS method cannot be applied in this case.

Recently, Slipchenko et al. demonstrated a new approach that combines the narrow CARS microscopy and Raman microspectroscopy. ${ }^{33}$ In this demonstration, CARS measurements at 2840 $\mathrm{cm}^{-1}$ were used for lipid imaging and Raman spectra were measured at several points of interest for further chemical identification. They report Raman spectral measurements from a lipid droplet (size $\sim 2 \mu \mathrm{m}$ ) inside a living cell with a CCD exposure time as short as $10 \mathrm{~ms} .{ }^{33}$ Note that Raman measurement with a CCD is a parallel process; i.e., all different frequency signals are measured simultaneously while our FMCARS spectroscopy is a serial process where only the signal at a single vibrational frequency is measured at a time. With the same total experiment time, both methods may show similar microspectroscopy performance with samples of strong vibrational signals such as lipid droplets. Chemical selectivity in imaging, however, is different. Since the FM-CARS method removes the nonresonant background and measures Raman-like vibrational signals in both imaging and microspectroscopy, we can perform fast beam-scanning imaging at multiple characteristic vibrational frequencies to obtain more information about the distributions of multiple chemical species. Microspectroscopy also greatly helps the image analysis, since it measures the same signals and enables us to choose the best vibrational frequencies for imaging.

\section{Conclusion}

The spectral focusing CARS method is advantageous over the narrow CARS one in terms of its simplicity, flexibility, and lower cost. ${ }^{19,20}$ It does not require any change of laser cavity or crystal temperature to switch the excitation vibrational frequency. We discuss several fundamental aspects of the spectral focusing CARS technique and report a new FM version of spectral focusing CARS microscopy that can generate Ramanlike CARS signals. Our method allows vibrational imaging at multiple frequencies in a reasonable time scale (a few seconds) and in situ microspectroscopy measurements. As we demonstrate in this work, the combination of ratio imaging and in situ 
microspectroscopy is a powerful tool for study of inhomogeneous samples. All the images in this work are obtained at the vibrational fingerprint region. This vibrational region has profound chemical information and can help to explore complicated biochemical processes in cells and tissues.

Acknowledgment. The authors gratefully acknowledge the Welch Foundation for the support of personnel (project F-1663). B.C.C. and S.H.L. also thank Dr. Payne Chang for providing the mouse ear used in this study.

\section{References and Notes}

(1) Evans, C.; Xie, X. S. Annu. Rev. Anal. Chem. 2008, 883.

(2) Evans, C. L.; Potma, E. O.; Puoris'hagg, M.; Cote, D.; Lin, C. P.; Xie, X. S. Proc. Natl. Acad. Sci. U.S.A. 2005, 102, 16807.

(3) Le, T. T.; Cheng, J. PLoS ONE 2009, 4, e5189.

(4) Cheng, J. X.; Xie, X. S. J. Phys. Chem. B 2004, 108, 827.

(5) Potma, E. O.; Jones, D. J.; Cheng, J. X.; Xie, X. S.; Ye, J. Opt. Lett. 2002, 27, 1168

(6) Lin-Vien, D.; Colthup, N. B.; Fateley, W. G.; Grasselli, J. G. The Handbook of Infrared and Raman Characteristic Frequencies of Organic Molecules; Academic Press: San Diego, 1991.

(7) Chen, B.-C.; Lim, S.-H. J. Phys. Chem. B 2008, 112, 3653. 9208.

(8) Dudovich, N.; Oron, D.; Silberberg, Y. J. Chem. Phys. 2003, 118,

(9) Lim, S.-H.; Caster, A. G.; Leone, S. R. J. Phys. Chem. B 2006, $110,5196$.

(10) Muller, M.; Schins, J. M. J. Phys. Chem. B 2002, 106, 3715.

(11) Cheng, J. X.; Book, L. D.; Xie, X. S. Opt. Lett. 2001, 26, 1341.

(12) Cheng, J. X.; Volkmer, A.; Book, L. D.; Xie, X. S. J. Phys. Chem. B 2001, 105, 1277 .

(13) Ganikhanov, F.; Evans, C.; Saar, B. G.; Xie, X. S. Opt. Lett. 2006, 31,1872 .

(14) Langbein, W.; Rocha-Mendoza, I.; Borri, P. Appl. Phys. Lett. 2009, 95, 081109.

(15) Rocha-Mendoza, I.; Langbein, W.; Watson, P.; Borri, P. Opt. Lett. 2009, 34, 2258.

(16) Saar, B. G.; Holtom, G. R.; Freudinger, C. W.; Ackermann, C.; Hill, W.; Xie, X. S. Opt. Exp. 2009, 17, 12532.

(17) Gershgoren, R.; Bartels, R. A.; Fourkas, J. T.; Tobey, R.; Murnane, M. M.; Kapteyn, H. C. Opt. Lett. 2003, 28, 361.
(18) Hellerer, T.; Enejder, A. M. K.; Zumbusch, A. Appl. Phys. Lett. 2004, 85,25 .

(19) Langbein, W.; Rocha-Mendoza, I.; Borri, P. J. Raman Spectrosc. 2009, 40, 800

(20) Pegoraro, A. F.; Ridsdale, A.; Moffatt, D. J.; Jia, Y.; Pezacki, J. P.; Stolow, A. Opt. Exp. 2009, 17, 2984.

(21) Rocha-Mendoza, I.; Langbein, W.; Borri, P. Appl. Phys. Lett. 2008, 93, 201103.

(22) Nibbering, E. T. J.; Wiersma, D. A.; Duppen, K. Phys. Rev. Lett. 1992, 68, 514 .

(23) Pegoraro, A. F.; Slepkov, A. D.; Ridsdale, A.; Lyn, R. K.; Moffatt, D. J.; Pezacki, J. P.; Thomas, B. K.; Fu, L.; Dong, L.; Ferman, M. E.; Stolow, A. Proc. SPIE 2010, 7569, 756908.

(24) Lee, S.; Zhang, D.; McCamant, D. W.; Kukura, P.; Mathies, R. A. J. Chem. Phys. 2004, 121, 3632.

(25) Mandel, L.; Wolf, E. Optical Coherence and Quantum Optics; Cambridge University Press: New York, 1995.

(26) Pestov, D.; Wang, X.; Ariunbold, G. O.; Murawski, R. K.; Sautenkov, V. A.; Dogariu, A.; Sokolov, A. V.; Scully, M. O. Proc. Natl. Acad. Sci. U.S.A. 2008, 105, 422.

(27) Vartiainen, E. M.; Rinia, H. A.; Muller, M.; Bonn, M. Opt. Exp. 2006, 14, 3622.

(28) Wurpel, G. W. H.; Muller, M. Chem. Phys. Lett. 2006, 425, 336.

(29) Sung, J.; Chen, B.; Lim, S.-H. J. Raman Spectrosc. 2010 (in press).

(30) Sung, J.; Chen, B.; Lim, S.-H. Opt. Lett. 2008, 33, 1404.

(31) Lim, S.-H.; Caster, A. G.; Leone, S. R. Phys. Rev. A 2005, 72, 041803.

(32) Beattie, J. R.; Bell, S. E. J.; Moss, B. W. Lipids 2004, 39, 407.

(33) Slipchenko, M. N.; Le, T. T.; Chen, H.; Cheng, J. J. Phys. Chem. B 2009, 113, 7681 .

(34) Fox, C. B.; Uibel, R. H.; Harris, J. M. J. Phys. Chem. B 2007, $111,11428$.

(35) Meier, R. J.; Csiszar, A.; Klumpp, E. J. Phys. Chem. B Lett. 2006, 110,5842 .

(36) Butler, M.; Salem, N.; Hoss, W.; Spoonhower, J. Chem. Phys. Lipids 1979, 29, 99.

(37) According to the manufacturer's label.

(38) Freudinger, C. W.; Min, W.; Saar, B. G.; Lu, S.; Holtom, G. R.; He, C.; Tsai, J. C.; Kang, J. X.; Xie, X. S. Science 2008, 322, 1857.

(39) Zeng, Y.; Saar, B. G.; Friedrich, M. G.; Chen, F.; Liu, Y.; Dixon, R. A.; Himmel, M. E.; Xie, X. S.; Ding, S. Bioenergy Res. 2010, 3, 272. (40) Gierlinger, N.; Schwanninger, M. Plant Physiol. 2006, 140, 1246.

(41) Fu, Y.; Wang, H.; Shi, R.; Cheng, J. X. Opt. Exp. 2006, 14, 3942.

(42) Cheng, J. X.; Volkmer, A.; Book, L. D.; Xie, X. S. J. Phys. Chem. B 2002, 106, 8493.

JP104553S 